\title{
14. VERZEICHNIS DER ABKÜRZUNGEN UND SIGLEN
}

A.

a. O.

Abb.

Adl.

Adr.

alttschech.

BAV

Bd., Bde.

Beih.

$\mathrm{Bl}$.

BSB, Cgm

bzw.

Clm

Cod.

Cod. Mare. lat.

Cod. Mell.

Cod. Pal. lat.

Cod. Tepl.

Cod. in scrin.

Cod. Ser. n.

CVP

Dipl. Arb.

Diss.

Dt. Akad. Wiss.

Ed.

Erg. Bd.

etc.

Fol.

hrsg.

Hs.

ital.

Kat.

mhd.

mittellat.

MS. Ambras.

N. F.

Nachdr.

$\mathrm{Nr}$.

ÖNB

$\mathrm{r}$
Anmerkung

am angegebenen Ort

Abbildung(en)

Adligat

Adresse

alttschechisch

Biblioteca Apostolica Vaticana

Band, Bände

Beiheft

Blatt, Blattes, Blätter

Bayerische Staatsbibliothek, Codex Germanicus Monacensis beziehungsweise

Codex latinus Monacensis $=$ BSB , Codex Latinus Monacensis

Codex

Codex Marcianus latinus $=$ Venedig, Marciana, Cod .

Codex Mellicensis = Melk, Stiftsbibl., Cod.

Codex Palatinus latinus $=$ Rom, BAV, Cod .

Codex Teplensis

Codex in scrinio $=$ Hamburg, Studienbibliothek, Cod. in scrin.

Wien, ÖNB, Codex Series nova

Codex Vindobonensis Palatinus $=$ Wien, ÖNB, Cod.

Diplomarbeit

Dissertation

Deutsche Akademie der Wissenschaften

Editor

Ergänzungsband

et cetera

Folium

herausgegeben

Handschrift

italienisch

Katalog

mittelhochdeutsch

mittellateinisch

Manuscriptum Ambrasiense

Neue Folge

Nachdruck

Nummer

Österreichische Nationalbibliothek

recto 
Rez.

S.

Sign.

Stiftsbibl

Taf.

u. a.

$\mathrm{v}$

$\mathrm{vgl}$.

Z.

zit.
Rezension

Seite

Signatur

Stiftsbibliothek

Tafel

und andere, unter anderem

verso

vergleiche

Zeile

zitiert 